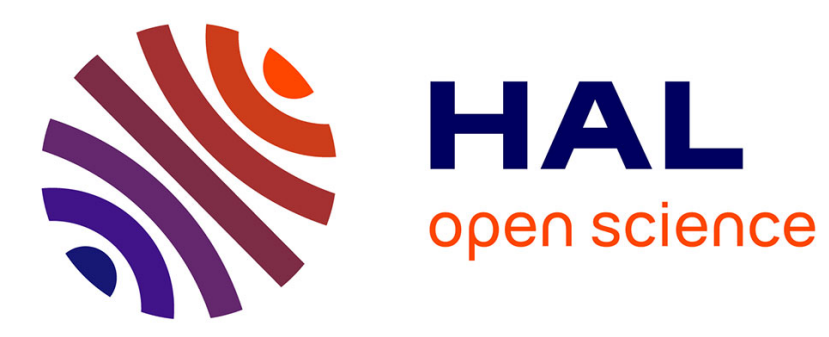

\title{
Complex Self-reproducing Systems
}

Roderick Edwards, Aude Maignan

\section{To cite this version:}

Roderick Edwards, Aude Maignan. Complex Self-reproducing Systems. Ali Sanayei, Ivan Zelinka, and Otto E. Rössler,. ISCS 2013: Interdisciplinarity Symposium on Complex Systems Emergence, Complexity and Computation, Springer, pp.65-76, 2014, Emergence, Complexity and Computation, 978-3-642-45437-0. 10.1007/978-3-642-45438-7_7 . hal-00953772

\section{HAL Id: hal-00953772 \\ https://hal.science/hal-00953772}

Submitted on 20 Mar 2014

HAL is a multi-disciplinary open access archive for the deposit and dissemination of scientific research documents, whether they are published or not. The documents may come from teaching and research institutions in France or abroad, or from public or private research centers.
L'archive ouverte pluridisciplinaire $\mathbf{H A L}$, est destinée au dépôt et à la diffusion de documents scientifiques de niveau recherche, publiés ou non, émanant des établissements d'enseignement et de recherche français ou étrangers, des laboratoires publics ou privés. 


\title{
Complex Self-reproducing Systems
}

\author{
Roderick Edwards ${ }^{1}$ and Aude Maignan ${ }^{2}$ \\ ${ }^{1}$ Department of Mathematics and Statistics, University of Victoria, P.O. Box 3060 STN CSC, Victoria, \\ B.C., Canada \\ ${ }^{2}$ Laboratoire Jean Kuntzmann, 51 rue des Mathematiques, B. P. 53, 38041 Grenoble Cedex 9 France
}

Keywords: self-reproducing systems, self-organizing systems, cellular automata, substitution systems, L-Systems.

\begin{abstract}
Cellular automata and L-Systems are well-known formal models to describe the behaviour of biological processes. They are discrete dynamical systems, each of which can have complex and varied behaviour. Here, we study a class of substitutive systems incorporating properties of both cellular automata and L-systems, that exhibits self-reproducing behaviour. A one-dimensional array of cells is considered, each cell has a set of modes or states which are determined by a number from $\mathbb{Z} / \mathbf{n} \mathbb{Z}^{*}$ ( $n$ prime). The behaviour of a cell depends on the states of its neighbours and obeys to an additive rule. It has also a cell-division mode, which allows the line of cells to grow. The behaviour of such a model can be complex, but, using algebraic techniques, we prove that it can describe a reproducing system.
\end{abstract}

\section{Introduction}

Biological processes [3,9] are often modelled by a continuous formalism in which the behaviour of the system is described by differential equations. The smooth variation of each variable is described as a function of others $[4,12,17]$. Another type of modelling uses a discrete formalism called multiagent formalism. Cellular Automata (CA) and L-Systems are parts of multi-agent systems which are homogeneous (all cells are identical). CA are defined by a lattice of sites, an alphabet of symbols and an evolution rule. Each cell evolves in discrete time steps according to some deterministic rules that depend only on local neighbours. In the configuration space, CA trajectories are likely to merge with time, and after many time steps, trajectories starting from almost all initial states become concentrated onto attractors. In these cases, completely disordered starting states evolve to more structured states by a process of self-organisation. In other cases, complex behaviour can appear. In his well-known papers [14,15], Stephen Wolfram proposed a classification of cellular automaton rules into four classes.

1. Class I: Evolution leads to a homogeneous state.

2. Class II: Evolution leads to a set of separated simple stable or periodic structures.

3. Class III: Evolution leads to a chaotic pattern.

4. Class IV: Evolution leads to complex localised structures, sometimes long-lived.

This classification is based on observations of typical behaviours. K. Sutner [10], but also F. Bagnoli and al. [2] and J.M. Baetens and al. [1] give a formalisation of those classes. For instance, the elementary rule 30 of Wolfram exhibits many different behaviours of class I,II,III and IV. On the other hand, an additive CA with fixed and periodic boundary conditions over a finite field $\mathbb{Z}_{\mathbf{n}}$ are more often of class I and II. This problem of determining dynamical possibilities for additive CA has been studied by Wolfram [13], who has obtained some powerful theoretical results thanks to algebraic tools.

Wolfram has also studied substitution systems [16], discrete dynamical systems such that, at every step, each site is replaced by a fixed block of new sites. Substitution systems are are clearly designed so that the number of sites can change. The theory of such systems was first developed by Aristid Lindenmayer and Przemyslaw Prusinkiewicz for modelling plant growth $[6,7]$, using a framework called 
L-systems. An L-system is defined as a t-tuple $G=(V, \omega, P)$ where $V$ is an alphabet, $\omega$ is the initial state of the system and $P$ is a set of production rules. One of the simplest and best-known examples is the modelling of the anabaena algae. The alphabet is reduced to two states $V=\{A, B\}$ and only two rules are required:

$$
\begin{array}{|l|l|}
A \rightarrow A B & B \rightarrow A \\
\hline
\end{array}
$$

At each step, each letter $A$ is transformed to the two letter sequence $A B$ and each letter $B$ is transformed to $A$. If the initial state is $A$, for example, we obtain the sequence $A B A A B A B A A B A A B A B A$ $A A B A B A$ after 6 steps. The family of possible transformation rules is huge and the study of the behaviours of L-systems is done on a case-by-case basis.

The aim of this paper is to combine properties of L-systems and additive CA in order to create a simple model which describes a self-reproducing "organism". The algebraic properties of this new model allow one to describe theoretically its behaviour, particularly its reproducing behaviour.

The model is described in Section 2. In Section 3 we present our analysis of its behaviour.

\section{Model description}

We consider a one dimensional structure in which the evolution of a particular site depends on its own value and those of its nearest neighbours. Sites are arranged around a circle (so as to give periodic boundary conditions). This structure is named a sequence. The number of sites of a sequence depends on time and is denoted by $N(t)$. The values of the sites of a sequence denoted $a$ also depend on time and are denoted by $a_{0}^{(t)}, \ldots, a_{N(t)-1}^{(t)}$. The possible site values are elements of a finite set $\mathbb{Z}_{\mathbf{n}}^{*}=\{\mathbf{1}, \mathbf{2}, \ldots, \mathbf{n}-\mathbf{1}\}$ ( $n$ prime). The value of a site of the neighbour-dependent substitution system we consider is computed by an operator $\Phi_{(\alpha, \beta)}$, which depends on two parameters $(\alpha, \beta) \in \mathbb{Z}_{\mathbf{n}}^{\mathbf{2}}$ and is defined by

- a simple additive rule of the form (taking the site index $i$ modulo $N(t)): a_{i}^{(t+1)}=\alpha a_{i-1}^{(t)}+\beta a_{i}^{(t)}+$ $\alpha a_{i+1}^{(t)}[\bmod n]$ if $\alpha a_{i-1}^{(t)}+\beta a_{i}^{(t)}+\alpha a_{i+1}^{(t)}[\bmod n] \neq 0$,

- and an expansive rule such that $a_{i}^{(t+1)}$ is divided into two sites of value 1 if $\alpha a_{i-1}^{(t)}+\beta a_{i}^{(t)}+$ $\alpha a_{i+1}^{(t)}[\bmod n]=0($ i.e., $0 \rightarrow 11)$.

We call such a system a Della Dora system, because it was first proposed to us by Jean Della Dora. The family of Della Dora systems is parameterised by $\alpha, \beta$ and $n$.

Subsequences are open in the sense that, by themselves, they carry no information about what is to either side. Thus we allow the possibility that a subsequence may be a full sequence, with periodic repetitions on either side, though it may, of course, be surrounded by something else.

Because of the expansive rule, the index of a site can change over time. But at a given step, a sequence may be represented by a characteristic polynomial $A^{(t)}(x)=\sum_{i=0}^{N(t)-1} a_{i}^{(t)} x^{i}$, and the additive rule can be represented by multiplication of the characteristic polynomial by a fixed Di-polynomial in $x$, namely $T(x)=\alpha x+\beta+\alpha x^{-1}$, according to $A^{(t+1)}(x)=T(x) A^{(t)}(x) \bmod \left(x^{N(t)}-1\right)$. This approach has been developed by Wolfram [13] for a pure additive CA. The new expansive rule modifies (but does not destroy) the algebraic properties of reference [13]. For instance, let us consider the commutative ring $\mathbb{Z}_{\mathbf{3}}$. The sequences built on $\mathbb{Z}_{\mathbf{3}}^{*}$ are composed of sites of value 1 or 2 . Six different dynamics can be defined by the six Di-polynomials $x+x^{-1}, 2 x+2 x^{-1}, x+1+x^{-1}, x+2+x^{-1}, 2 x+1+2 x^{-1}$, and $2 x+2+2 x^{-1}$. We present 3 examples which correspond to 3 different behaviours.

Example 1. First, consider the Di-polynomial $T(x)=x+2+x^{-1}$ on $\mathbb{Z}_{\mathbf{3}}^{*}$. The sites evolve such that, at each time step, the value of a site $a_{i}^{(t+1)}$

- takes the value $a_{i-1}^{(t)}+2 a_{i}^{(t)}+a_{i+1}^{(t)}[\bmod 3]$ if this value is not equal to 0 ; 
- else, splits into two sites, both of which take the value 1.

This is a specific L-system and can be defined using this following transformation rules, where the site in brackets is modified according to the rule defined by the arrow.

$$
\begin{array}{|l|l|l|}
\hline 1\langle 1\rangle 1 \rightarrow 1 & 1\langle 1\rangle 2 \rightarrow 2 & 1\langle 2\rangle 1 \rightarrow 11 \\
1\langle 2\rangle 2 \rightarrow 1 & 2\langle 1\rangle 1 \rightarrow 2 & 2\langle 1\rangle 2 \rightarrow 11 \\
2\langle 2\rangle 1 \rightarrow 1 & 2\langle 2\rangle 2 \rightarrow 2 & \\
\hline
\end{array}
$$

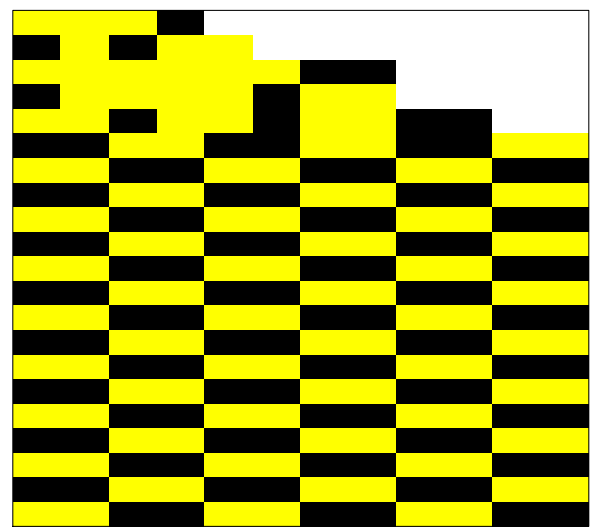

Fig. 1. Example of synchronisation: $T(x)=x+2+x^{-1}$ over $\mathbb{Z}_{3}^{*}$ with substitution rule $0 \rightarrow 11$.

The evolution of this system can be represented graphically at successive time steps by successive lines. Sites with value one are represented by a light colour; sites with value two are dark.

Figure 1 shows the system's behaviour when the initial configuration is (1112). For every initial configuration, $N(t)$ is bounded and the system converges to a fixed point or a cycle.

This example thus belongs to Wolfram's class $I I$ and describes a synchronisation phenomenon. This synchronisation is a strong case of a self-organizing process where a global coordination arises out of an initially disordered system.

The behaviour of the second example is more complex.

Example 2. Consider the system with Di-polynomial $T(x)=2 x+1+2 x^{-1}$ on $\mathbb{Z}_{3}^{*}$ and the $0 \rightarrow 11$ rule.

This is defined in detail by the following L-system :

$$
\begin{array}{|l|l|l|}
\hline 1\langle 1\rangle 1 \rightarrow 2 & 1\langle 1\rangle 2 \rightarrow 1 & 1\langle 2\rangle 1 \rightarrow 11 \\
1\langle 2\rangle 2 \rightarrow 2 & 2\langle 1\rangle 1 \rightarrow 1 & 2\langle 1\rangle 2 \rightarrow 11 \\
2\langle 2\rangle 1 \rightarrow 2 & 2\langle 2\rangle 2 \rightarrow 1 & \\
\hline
\end{array}
$$

Figure 2 shows the system's behaviour when the initial configuration is (1112111). The behaviour is complicated and there is no obvious nested structure, so it belongs to Wolfram's class III.

Between the above examples, there exists a class of dynamics where the behaviour is both rich and predictable. 


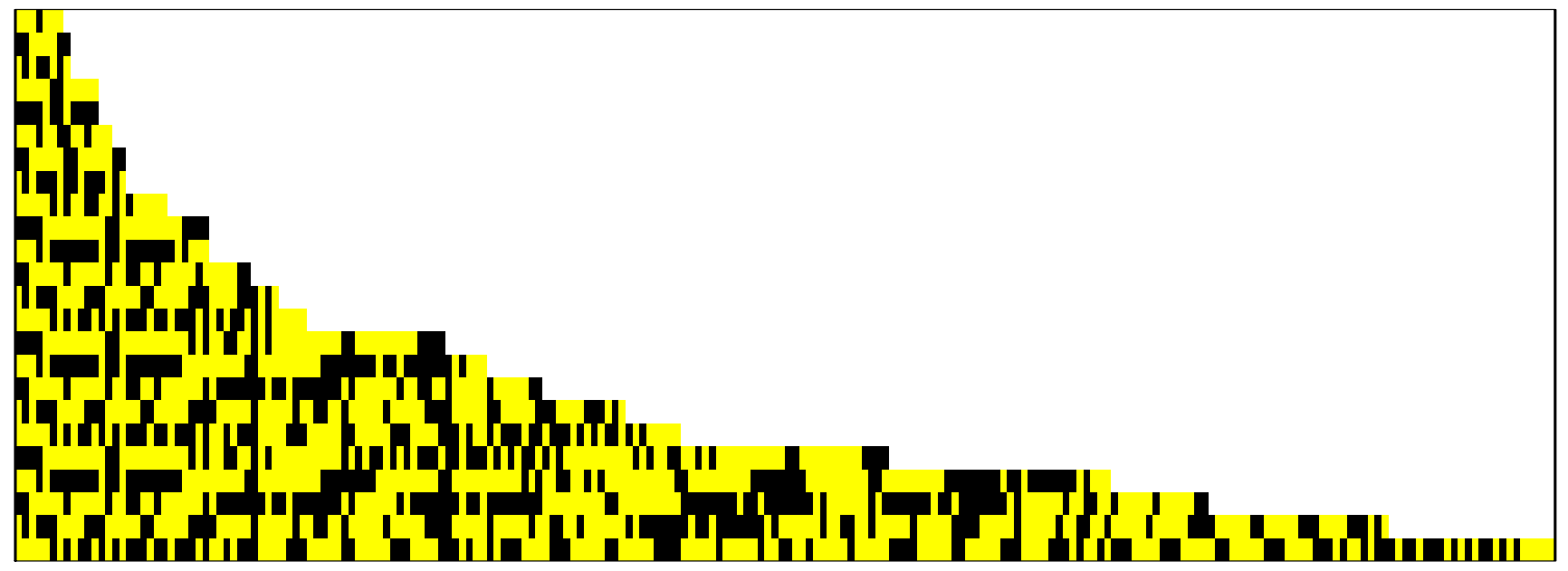

Fig. 2. Example of chaotic behaviour : $T(x)=2 x+1+2 x^{-1}$ over $\mathbb{Z}_{\mathbf{3}}^{*}$, with substitution rule $0 \rightarrow 11$.

Example 3. Consider the system with Di-polynomial $T(x)=2 x+2+2 x^{-1}$ on $\mathbb{Z}_{\mathbf{3}}^{*}$, again with the rule $0 \rightarrow 11$.

The transformation rule is described in the following table:

$$
\begin{array}{|l|l|l|}
\hline 1\langle 2\rangle 1 \rightarrow 2 & 1\langle 2\rangle 2 \rightarrow 1 & 1\langle 1\rangle 1 \rightarrow 11 \\
1\langle 1\rangle 2 \rightarrow 2 & 2\langle 2\rangle 1 \rightarrow 1 & 2\langle 2\rangle 2 \rightarrow 11 \\
2\langle 1\rangle 1 \rightarrow 2 & 2\langle 1\rangle 2 \rightarrow 1 & \\
\hline
\end{array}
$$

The behaviour is complicated but nested structure can be identified. Moreover nested structures give birth to new nested structures. This belongs to Wolfram's class $I V$.

More generally, the discrete phase portrait of such systems is difficult to analyse because the dimension of the phase space evolves over time. Nevertheless, algebraic tools bring powerful results. In Example 3 above, the open subsequences $11 \ldots 11=1^{k}$ with $k \geq 4$ always produce a subsequence of $1 \mathrm{~s}$ of at least the same length after each time step (when $k$ is exactly 4 , the subsequence of 1 s remains the same length: the open subsequence $21^{4} 2$ produces another sequence containing $21^{4} 2$ ). This property is at the root of a very interesting type of behaviour: self-replication. We have, in fact, that

Proposition 1. $a_{i-1}^{(t)}=a_{i}^{(t)}=a_{i+1}^{(t)}=1$ implies that $a_{i}^{(t+1)}$ is transformed into two sites of value 1 if and only if 1 is a root of its Di-polynomial.

So in $\mathbb{Z}_{\mathbf{n}}(n>2), \Phi_{(\alpha, \beta)}$ gives behaviours of Wolfram class $I V$ if $(x-1) \mid x\left(\alpha x+\beta+\alpha x^{-1}\right)$. Equivalently, $\Phi_{(\alpha, \beta)}$ gives behaviours of Wolfram class $I V$ if $2 \alpha+\beta=0[\bmod n]$. We call systems with this property, $2 \alpha+\beta=0[\bmod n]$, Della Dora systems of type 1 .

Every configuration in a Della Dora system of type 1 has a unique successor at every time step. However, a configuration may have several distinct predecessors. $\alpha x^{2}+\beta x+\alpha$ is not prime and $\Phi_{(\alpha, \beta)}$ is not injective so these dynamics are irreversible.

The following section describes the properties of Della Dora systems of type 1. We will see how the behaviours of these systems can be described by a finite number of subsequences. This corresponds 


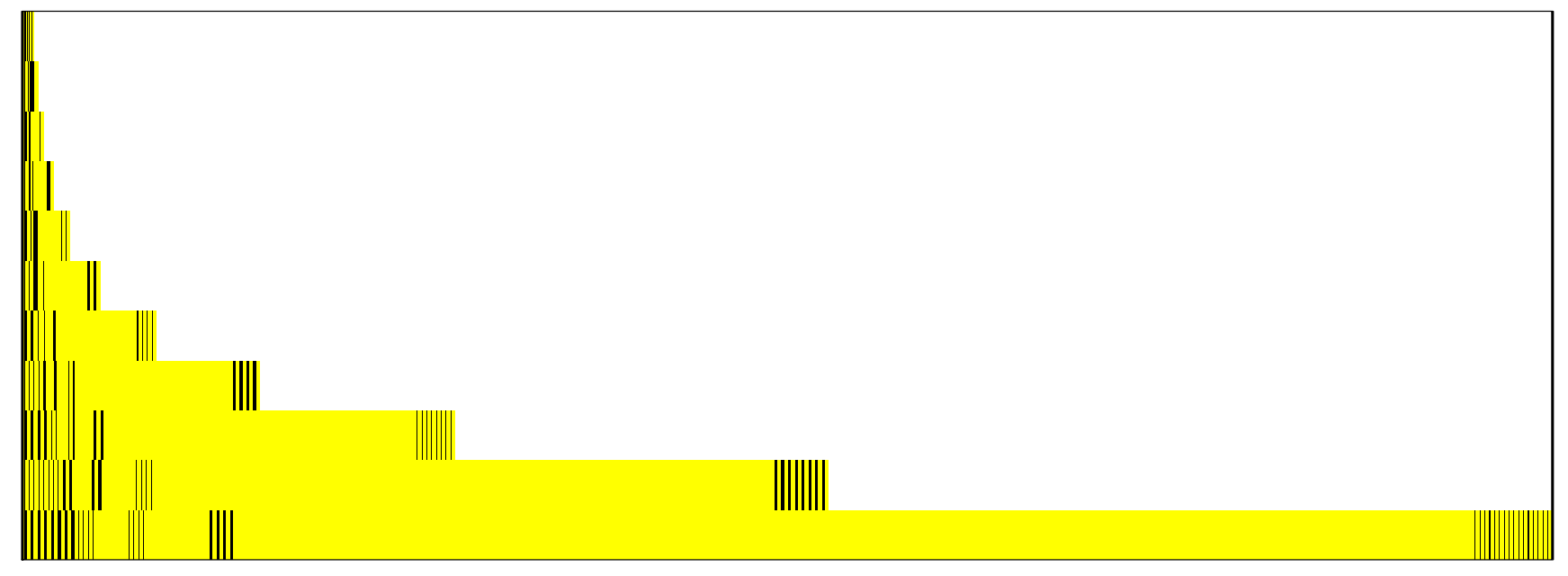

Fig. 3. Example of self replication: $T(x)=2 x+2+2 x^{-1}$ over $\mathbb{Z}_{3}^{*}$, with substitution rule $0 \rightarrow 11$.

to a self-organizing phenomenon. We will also see that over time, specific subsequences give birth to a copy of themselves. This corresponds to a self-reproducing phenomenon.

\section{Analysis of Della Dora systems of type 1}

\subsection{First definitions}

Cycles in phase space can be determined by a polynomial approach. A $P$-periodic cycle of an additive $\mathrm{CA}$ is a list of $P$ sequences, each of length $N$,

$$
\left(a^{(t)}, a^{(t+1)}, \ldots, a^{(t+P-1)}\right),
$$

such that their corresponding characteristic polynomials, $A^{(t+i)},(i \in\{0,1, \ldots P-1\})$ obey

$$
A^{(t+i+1)}(x)=T(x) A^{(t+i)}(x) \bmod \left(x^{N}-1\right)
$$

and

$$
A^{(t+i)}(x)=T(x)^{P} A^{(t+i)}(x) \bmod \left(x^{N}-1\right) .
$$

The cycles of the dynamical system $\Phi_{(\alpha, \beta)}$ are the cycles of the additive dynamics defined by the Di-polynomial $T_{(\alpha, \beta)}$, such that every sequence occurring in the cycle has no null sites (a 0 site would lead to a splitting $0 \rightarrow 11$ and thus could not be part of a cycle).

For Example 3, it is possible to give a formal proof showing that there are only two types of cycles, one of periodicity $P=1$ and another of periodicity $P=2$. The cycles of periodicity 2 can be denoted by $\left((2211)^{k},(1122)^{k}\right)$ where $k$ is an integer and $(2211)^{k}$ and $(1122)^{k}$ are the successive states of the cycle $\left(\right.$ i.e., $\Phi_{(2,2)}\left((2211)^{k}\right)=(1122)^{k}$ and $\left.\Phi_{(2,2)}\left((1122)^{k}\right)=(2211)^{k}\right)$.

Here, $(2211)^{k}$ denotes a sequence of length $4 k$ such that $(2211)^{k}=22112211 \ldots 2211$. With the same notation, the cycles of periodicity 1 can be denoted by $\left((21)^{k}\right)$. All these cycles are reached only by 
themselves. In general, it is possible also to have transient approaches to cycles. An example in the system with $\alpha=2, \beta=1$ and $n=5$ (a Della Dora system of type 1 ) is the sequence (141) for which $\Phi_{(2,1)}(141)=(131)$ which is then part of the cycle $((131),(424))$.

Over time, an open subsequence $11 \ldots 11=1^{k}(k \geq 4)$ produces a subsequence of 1 s with length that grows (exponentially) without bound when $k>4$, and with length that may remain fixed or may grow when $k=4$, with no further variation in behaviour. If we consider these as sites in a 'base state,' then information resides in regions in which some 'not-1' sites occur. The characteristic sequences of a sequence $a$ are maximal open subsequences of sites of $\mathbb{Z}_{\mathbf{n}}$ which do not contain $1^{k}$ with $k \geq 4$ and neither begin nor end with a 1 . In effect, the occurrence of expanding subsequences of 1 s separate the characteristic sequences, and the subsequence 1111 can be considered a separator: if $b$ and $c$ are two characteristic sequences of a sequence $a$, changing the values of any sites of $b$ will never affect values of sites of $c$. Characteristic sequences $b$ and $c$ evolve independently. They can have periodic behaviour, they can vanish, and they can also grow. If 1111 appears at some time step from the interior of a characteristic sequence, it creates new independent subsequences. This is, in that case, a reproduction process.

A sequence containing the subsequence 1111 we call a broken sequence; otherwise it is unbroken. Broken sequences consist of one or more characteristic sequences separated by separators of the form $1^{k}$ with $k \geq 4$. Unbroken sequences cannot be considered characteristic sequences, since they do not have 1111 to either side.

\subsection{Dynamics of characteristic sequences}

The dynamics of a characteristic sequence (which is open) is sightly different from the dynamics of a (closed) sequence. Let $\Phi_{o}$ denote this new dynamic applied to an open sequence $s=s_{0} \ldots s_{N-1}$ surrounded by multiple sites with value 1 . Then,

$\Phi_{o}(s)=\alpha+\beta+\alpha s_{0}, \alpha+\beta s_{0}+\alpha s_{1}, \ldots, \alpha s_{i-1}+\beta s_{i}+\alpha s_{i+1}, \ldots, \alpha s_{N-2}+\beta s_{N-1}+\alpha, \alpha s_{N-1}+\beta+\alpha$,

where, as before, we apply the expansive rule $0 \rightarrow 11$ where applicable. The new computation of the extremal sites is a consequence of the fact that $s$ is bordered by sites of value 1 . The independent evolution of characteristic sequences can now be expressed by $\Phi\left(b 1^{*} c 1^{*}\right)=\Phi_{o}(b) 1^{*} \Phi_{o}(c) 1^{*}$ where $\left(b 1^{*} c 1^{*}\right)$ is a closed sequence constituted by two characteristic sequences, and $*$ is a non specified integer greater than or equal to 4 . It is convenient to introduce a more compressed notation: $\Phi_{o}(b ; c)=$ $\Phi_{o}(b) ; \Phi_{o}(c)$. This notation can easily be extended to more than two characteristic sequences.

For Example 3, using this new notation, $\Phi_{o}^{2}(2)=\Phi_{o}(222)=2 ; 2$. So, when the characteristic sequence (2) appears, after two steps it splits into two characteristic sequences (2). The periodicity of reproduction of the characteristic sequence (2) is therefore 2.

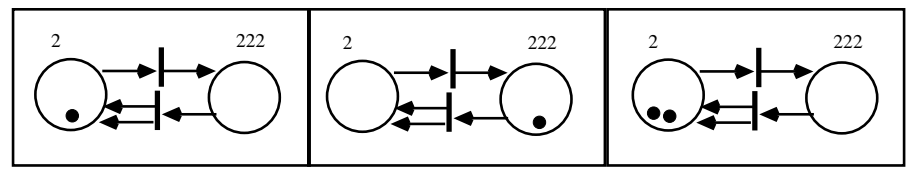

Fig. 4. Petri net of the characteristic sequence (2) at time $t, t+1$ and $t+2$ under the dynamics of the system with Di-polynomial $T=2 x^{2}+2 x+2$ over $\mathbb{Z}_{3}^{*}$.

Figure 4 represents the Petri net of this simple example of behaviour. More complex examples can be represented in this way. For instance, let us consider again the dynamics $\Phi_{(2,1)}$ over $\mathbb{Z}_{\mathbf{5}}^{*}$. Figure 5 shows the Petri net associated with the characteristic sequence 233213. 


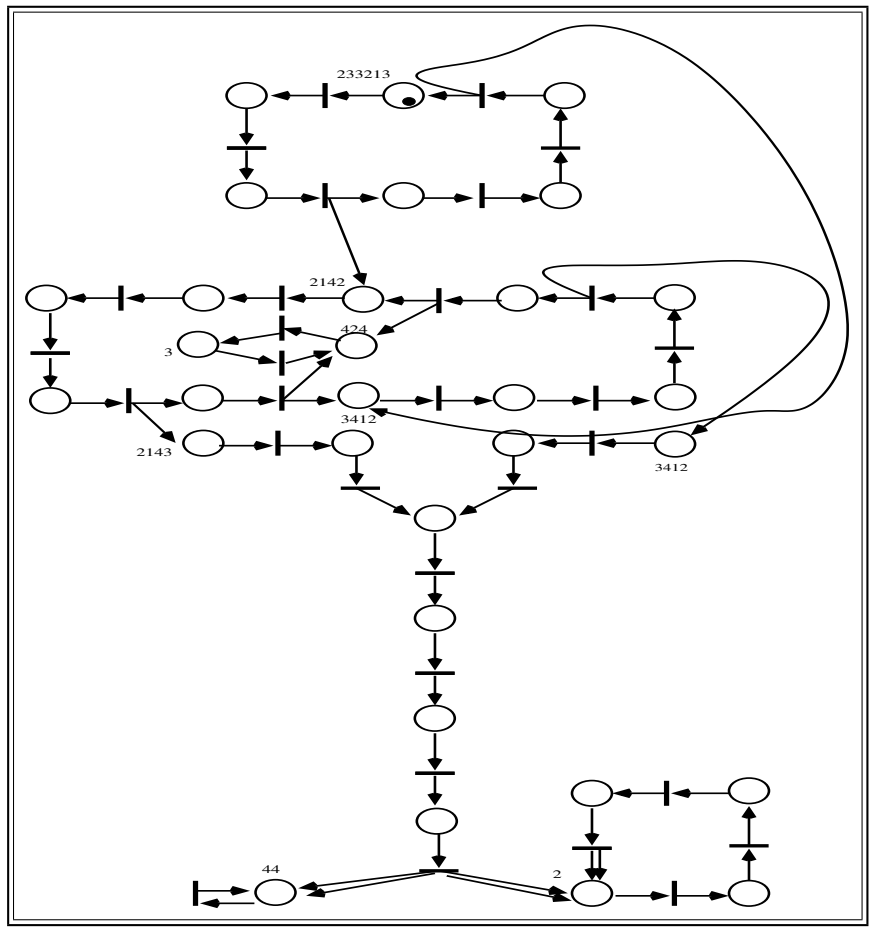

Fig. 5. Petri net for the characteristic sequence (233213) under the dynamics of the system with Di-polynomial $T=2 x^{2}+x+2$ over $\mathbb{Z}_{\mathbf{5}}^{*}$. 
The same characteristic sequences can appear several times in a sequence. Let $\mathcal{S}_{a}$ denotes the set of distinct characteristic sequences that are contained in the sequence $a$.

For Example 3, it is easy to show that $1^{k}(k \in \mathbb{N}$ and $k \geq 4)$ are the unique separators. We can consider 1111 a weak separator, i.e., a separator that persists but whose length does not increase. Two characteristic sequences that are separated by a weak separator correspond to independent entities which stay linked geographically. For example, $\Phi_{(2,2)}\left(21^{4} 2\right)=\left(121^{4} 21\right)$ and the sequence of 1 's does not grow longer. On the other hand, if the separator is not weak, the characteristic sequences move apart.

\subsection{Behaviour analysis}

For all Della Dora systems of type 1 we have studied, with various choices of $\alpha, \beta$ and $n$, we have proven that the behaviour of any sequence that does not belong to a cycle, and is not on a transient leading to a cycle, can be described by a finite number of characteristic sequences.

More precisely, let $a$ be the initial state. Even if the sequence continually grows, there exists a finite set of characteristic sequences $\mathcal{S}$ depending on $a$, such that for all integers $l, \mathcal{S}_{\Phi^{l}(a)} \subset \mathcal{S}$. In order to prove this strong property, new terms have to be defined.

Broken sequences grow without bound: $\lim _{t \rightarrow \infty} N(t)=\infty$. This is true even if there is only one separator and it is weak, because, even though the length of the separating sequence may remain fixed, it feeds new sites into the characteristic sequence at either side. Unbroken sequences may break and then grow without bound, or may not, such as is the case when they fall into a cycle, possibly after a transient. Of course, if a sequence grows without bound, it cannot be part of a cycle or a transient leading to a cycle, and if a sequence does not grow without bound, it must reach a cycle because the accessible state space is then finite. This still leaves open the possibility, a priori, of an unbroken sequence that never breaks but grows without bound. In order to deal with both unbroken sequences and characteristic sequences, and in particular to investigate whether or not they eventually split, we consider the internal dynamics of an arbitrary subsequence.

Let $\Phi_{r}$ denote the partial evaluation of an open sequence $s$ such that:

$$
\Phi_{r}(s)=\alpha s_{0}+\beta s_{1}+\alpha s_{2}, \ldots, \alpha s_{i-1}+\beta s_{i}+\alpha s_{i+1}, \ldots, \alpha s_{N-3}+\beta s_{N-2}+\alpha s_{N-1}
$$

where the expansive rule $0 \rightarrow 11$ is once again applied. Note that $\Phi_{r}(s)$ does not transform the end sites of the open sequence $s$, because that would require information on sites outside of $s$.

We say that a subsequence $s$ intrinsically separates if there exists a number of steps $k$ such that $\Phi_{r}^{k}(s)$ contains a separator. When a subsequence separates intrinsically, the value of the first site is never influenced by the value of the site which is just after the subsequence.

Unbroken sequences that eventually cycle clearly do not separate (break). Characteristic sequences can also cycle in the sense that, using our condensed notation, $\Phi_{o}^{P}(s)=s$ and these also clearly do not separate. An example, in the system with $\alpha=2, \beta=1$ and $n=5$, is $s=3$ for which $\Phi_{o}^{2}(3)=\Phi_{o}(424)=3$. Characteristic sequences can also be on transients leading to cycles, such as $s=4$ in the above example, for which $\Phi_{o}(4)=3$. If the characteristic sequence $s$ cycles, there exists an integer $i_{s}\left(0 \leq i_{s} \leq 3\right)$ such that $\left(s 1^{i_{s}}\right)^{k}$ also cycles, but $\gamma\left(s 1^{i_{s}}\right)^{k}\left(\gamma \in \mathbb{Z}_{\mathbf{n}}\right)$ does not necessary cycle any more. For instance $(424)^{10}$ cycles but $4(424)^{10}$ does not cycle and does not separate intrinsically. The cyclic part of $\Phi_{o}^{t}\left(4(424)^{10}\right)$ decreases when $t$ increases and $\Phi_{o}^{10}\left(4(424)^{10}\right)=31124333114 ; 3 ;(424)^{8}$. In other examples, the cyclic part can also totally disappear. We call a disturbed cycle a characteristic sequence of the form $*\left(s 1^{i_{s}}\right)^{k} *$ where $s$ is a cycle and $*$ represents any subsequence of $\mathbb{Z}_{\mathbf{n}}$ that does not contain a separator, and is small enough such that $*\left(s 1^{i_{s}}\right)^{k} *$ does not separate intrinsically. $*$ can also be the empty set, for instance, $s=11211211324434434423$ is such that $\Phi_{(2,1)}^{5}(s)=s$ but $\Phi_{o}^{5}(s)=3 ; 212111221112113244344343112$ and $s$ is already a disturbed cycle.

Our objective, then, is to show that every subsequence above a certain size either separates intrinsically or is a transient to a disturbed cycle, or eventually cycles, whether considered as an unbroken 
sequence or as a characteristic sequence. In the case of intrinsically separable subsequences, even if, over the $k$ steps required to guarantee separation, some of the resulting characteristic sequences became longer, there is still a finite size to which they can grow in $k$ steps. In the case of disturbed cycles, we have observed that there exists a finite small integer $\lambda$ such that for all integers $l>\lambda$, the number of attached cycles $s$ of $\Phi_{o}^{l}\left(*\left(s 1^{i_{s}}\right)^{k} *\right)$ decreases or remains the same and there exists an integer $\hat{l}$ such that $\Phi_{o}^{\hat{l}}\left(*\left(s 1^{i_{s}}\right)^{k} *\right)$ is composed of intrinsically separable subsequences and cycles. Thus, over time, a disturbed cycle disappears and generates a set of characteristic sequences. The size of those characteristic sequences is bounded and the set is finite.

This will provide a proof that the number of characteristic sequences generated by any initial sequence is bounded. Consequently, for any given initial broken sequence, the entire future behaviour can be described by a finite number of characteristic sequences.

We have developed an $R$ program whose arguments are $n$ of the finite set $\mathbb{Z}_{\mathbf{n}}^{*}, \alpha$ and $\beta$ of the dynamics and a length $N$ for subsequences. The program computes the list of all the subsequences of length $N$ that do not separate intrinsically using the separator 1111.

For Example 3, running our $R$ program shows that every subsequence of length at least 18 either intrinsically separates or is a disturbed cycle or eventually cycles.

Thus, the behaviour of every broken sequence can be defined by a finite set of characteristic sequences.

More generally, we conjecture that for any Della Dora system of type 1, there exists a length $N$ such that every characteristic sequence or unbroken sequence of length $N$

- intrinsically separates

- or is a transient to a disturbed cycle or a cycle

- or eventually cycles.

This will imply that the entire future behaviour of every sequence can be described by a finite number of unbroken sequences and a finite number of characteristic sequences and the separators. Finally the evolution of any sequence would be representable by a finite Petri net.

\section{Conclusion}

Cellular automata are capable of very complex behaviour. They model a large range of biological phenomena. For instance, Wolfram has used them to describe natural systems from snowflakes to mollusc shells. The class of Della-Dora systems are less simple in construction than cellular automata and can also have very complex behaviour. The idea is to add the possibility of modelling the important biological process of mitosis. In this extended abstract, we have focussed on the study of a subclass of such systems, which we call Della-Dora systems of type 1 . These are irreversible systems that give birth to very interesting self-organizing and self-reproducing behaviours. In future work, the behaviour of Della-Dora system of type 1 will be explored as a simulation of the type of behaviour seen in algae or filaments in a Petri dish. A formalisation for the classification of Della-Dora systems of type 1 will also be explored. For Cellular Automata, quantitative measures have been proposed to identify the Wolfram classes. In our case, the number of sites evolves and these measures must be adapted.

\section{References}

1. J. M. Baetens and B. De Baets. Phenomenological study of irregular cellular automata based on Lyapunov exponents and Jacobians. Chaos, 20,2010.

2. F. Bagnoli, R. Rechtman, and S. Ruffo, Damage Spreading and Lyapunov Exponents in Cellular Automata, Physics Letters A, 172, 1992, pp. 3438.

3. D. Barnes, D. Chu, Introduction to Modelling for Biosciences. Springer Verlag,2010. 
4. J. R. Junck. Ten Equations that Changed Biology: Mathematics in Problem-Solving Biology Curricula, Bioscene, 1997.

5. P. Favati , G. Lotti, L. Margara, Additive one-dimensional cellular automata are chaotic according to Devaney definition of chaos, Theoretical Computer Science A, 174 (1-2) (1997), 157-170.

6. A Lindenmayer, Mathematical models for cellular interaction in development (Parts I and II), Journal of Theoretical Biology, 18:280-315, 1968

7. P Prusinkiewicz, A Lindenmayer, The algorithmic beauty of plants. Springer-Verlag

8. P Prusinkiewicz, Marc Hamel, Eric Mjolsness, Animation of Plant Development, Proceedings of SIGGRAPH 93 (Anaheim, California, August 1-6) In Computer graphics Proceedings, Annual Conference Series, 1993, ACM SIGGRAPH, pp 351-360.

9. J.D. Murray, Mathematical Biology. Springer-Verlag, 3rd ed. in 2 vols.: Mathematical Biology: I. An Introduction, 2002; Mathematical Biology: II. Spatial Models and Biomedical Applications, 2003.

10. K. Sutner, Classification of cellular automata, Encyclopedia of Complexity and System Science, ed. R. A. Meyers, Springer 2009.

11. K. Sutner, Classifying circular cellular automata, Physica D, 45 (1990), pp. 386395

12. P. Turchin, Evolution in population dynamics. Nature 424,2003, pp 257258.

13. O. Martin, A. M. Odlyzko, S. Wolfram, Algebraic Properties of Cellular Automata, Communications in Mathematical Physics, Springer-Verlag, 1984.

14. S. Wolfram, Computation theory of cellular automata. Comm. Math. Physics, 96(1);15-57,1984.

15. S. Wolfram, Universality and complexity in cellular automata. Physica 10D (1984) 1-35.

16. S. Wolfram, A New Kind of Science, Wolfram Media,2002. http://www.ics.uci.edu/ eppstein/ca/wolfram.html

17. T. Yoshida, N.G. Hairston and S.P. Ellner (2004) Evolutionary tradeoff between defence against grazing and competitive ability in a simple unicellular alga, Chlorella vulgaris. Proc. R. Soc. Lond. B 271, pp19471953, 2004. 